\section{Robust Stabilization of a Nonlinear Cement Mill Model}

F. Grognard, F. Jadot, L. Magni, G. Bastin, R. Sepulchre, and V. Wertz

Abstract-Plugging is well known to be a major cause of instability in industrial cement mills. A simple nonlinear model able to simulate the plugging phenomenon is presented. It is shown how a nonlinear robust controller can be designed in order to fully prevent the mill from plugging.

Index Terms-Cement mill, plugging, robust state feedback, semiglobal stabilization, saturation.

\section{INTRODUCTION}

This note is concerned with the feedback stabilization of industrial cement mills. A schematic layout of a typical cement milling circuit is shown in Fig. 1. The plant is made up of the interconnection of a ball mill and a separator. The ball mill is fed with raw material (cement clinker). After grinding, the milled material is introduced in the separator where the finished product (i.e., the particles that are small enough) is separated from the oversize particles (also called tailings) which are recycled to the ball mill.

Traditionally, the application of feedback control to cement mills is limited to monovariable classical PI regulation of the circulating load (the tailings flow rate) with either the feed flow rate or the separator speed as control action. Recently, linear multivariable control techniques have been introduced to improve the performances of the milling circuit (see, e.g., [1], [2] and the references therein). However linear controllers based on a linear approximation of the process, are stable and effective only in a limited range around the nominal operating point. On some occasions, it is observed on real plants that intermittent disturbances (like for instance changes in the hardness of the raw material) may drive the mill to a region where the controller cannot stabilize the plant. This is well known by the operators as the so-called plugging phenomenon of ball mills.

In order to cope with this problem, a simple nonlinear model of the milling circuit has been presented in [4]. This model specifically includes the mill load (amount of material inside the mill) as a state variable. It is able to reproduce the plugging phenomenon in a realistic way. In [5], the authors also observe that this state variable is essential for a better control of the process.

In [4], a state feedback controller based on this nonlinear model is presented. It is build on a nonlinear predictive control strategy and, as such, is really the extension of the previous linear quadratic channel (LQG) controller of [1]. With this controller, the nominal performance of the closed loop system is improved but the robust stability is not guaranteed. The system has a larger stability region than with the linear

Manuscript received February 2, 1999; revised March 5, 2000. Recommended by Associate Editor A. Rantzer. This work was supported by the Belgian Program on Interuniversity Attraction Poles, initiated by the Belgian State, Prime Minister's Office for Science, Technology and Culture. F. Grognard is Aspirant FNRS.

F. Grognard, G. Bastin, and V. Wertz are with the Centre for Systems Engineering and Applied Mechanics (CESAME), Université Catholique de Louvain,

B-1348 Louvain-la-Neuve, Belgium (e-mail: bastin@csam.ucl.ac.be).

F. Jadot is with Schneider, 92500 Rueil Malmaison, France.

L. Magni is with the Dipartimento di Informatica e Sistemistica, Universitá di Pavia, 27100 Pavia, Italy.

R. Sepulchre is with the Institut Montefiore, B28, Université de Liège, 4000

Liège, Belgium.

Publisher Item Identifier S 0018-9286(01)03445-6.

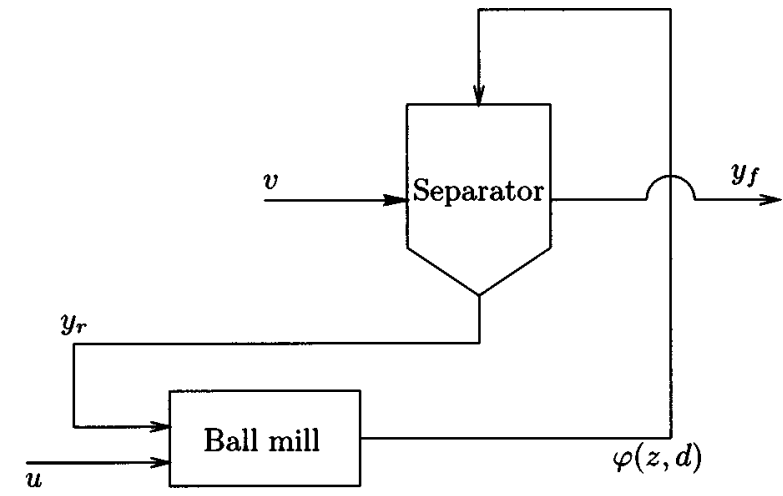

Fig. 1. Schematic view of a milling circuit.

controller of [1], but the risk of mill plugging is nevertheless not fully avoided.

The contribution of this note is then to describe a specific state feedback nonlinear controller such that the system has a single equilibrium semiglobally stable (and therefore globally attracting) in the domain of physical existence of the process. The controller is therefore able to completely prevent the mill from plugging. Moreover this controller is robust because it requires very little a priori knowledge of the plant (only the shapes of the grinding and separation functions need to be known).

\section{MATHEMATiCAl Modeling}

The following notations are introduced (see Fig. 1). The mill is fed with cement clinker at a feeding rate $u$ [tons $/ \mathrm{h}]$. The separator is driven by its rotational speed $v[\mathrm{rpm}]$. The tailings are recycled at a rate $y_{r}$ $[\mathrm{t} / \mathrm{h}]$ to the mill while the finished product exits the plant at a rate $y_{f}$ $[\mathrm{t} / \mathrm{h}]$. The plant is described by a simple dynamical model with three state variables

$$
\begin{aligned}
T_{f} \dot{y}_{f} & =-y_{f}+(1-\alpha(v)) \varphi(z, d) \\
T_{r} \dot{y}_{r} & =-y_{r}+\alpha(v) \varphi(z, d) \\
\dot{z} & =-\varphi(z, d)+y_{r}+u
\end{aligned}
$$

where $T_{f}, T_{r}[\mathrm{~h}]$ are time constants, $z[\mathrm{t}]$ is the amount of material in the mill (also called the mill load), $d$ represents the clinker hardness, $\alpha(v)$ is the separation function and $\varphi(z, d)$ is the ball mill outflow rate.

The grinding function $\varphi(z, d)$ is shown in Fig. 2 for different values of $d$. It is a non monotonic function of the mill load $z$. When $z$ is too high, the grinding efficiency decreases and leads to the obstruction of the mill (plugging). A low value of $z$ is also undesirable because it causes a fast wear of the balls.

The separation function $\alpha(v)$, shown in Fig.3, is a monotonically increasing function of the rotational speed $v$ of the separator, constrained between 0 and 1 with $0 \leq v \leq v_{\max }$ and $\alpha\left(v_{\max }\right)<1$.

Of paramount importance is the fact that with this modeling of the grinding and separation functions, the system (1)-(3) is positive (see, e.g., [3]) in accordance with the physical reality:

$$
\begin{aligned}
& \text { if } y_{f}(0) \geq 0, \quad y_{r}(0) \geq 0, \quad z(0) \geq 0, \quad u(t) \geq 0 \\
& \text { and } 0 \leq v(t) \leq v_{\max } \text { for all } t \geq 0 \\
& \text { then } y_{f}(t) \geq 0, \quad y_{r}(t) \geq 0
\end{aligned}
$$

and $z(t) \geq 0$ for all $t \geq 0$. Indeed, (1)-(3) show that whenever a component of the state becomes zero, its derivative is nonnegative. 


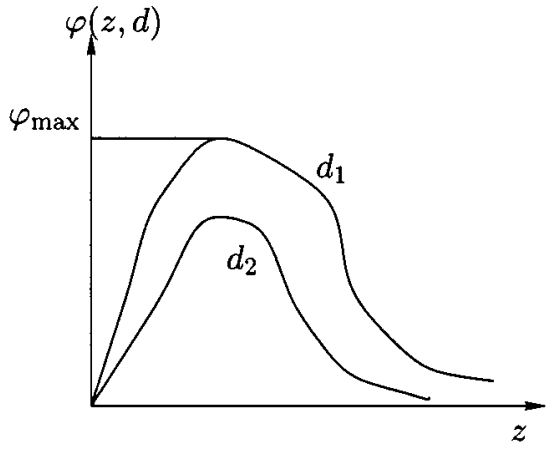

Fig. 2. Grinding function.

\section{STABILITY OF EQUILIBRIA}

Assuming that the clinker hardness $d$ is constant, the equilibria of the system $\bar{y}_{f}, \bar{y}_{r}, \bar{z}$ are parametrized by the constant inputs $\bar{u}$ and $\bar{v}$

$$
\begin{aligned}
\bar{y}_{f} & =\bar{u} \\
\bar{y}_{r} & =\frac{\alpha(\bar{v}) \bar{u}}{1-\alpha(\bar{v})} \\
\varphi(\bar{z}, d) & =\frac{\bar{u}}{1-\alpha(\bar{v})} .
\end{aligned}
$$

In view of the shape of $\varphi(z, d)$, as illustrated in Fig.4, there may be zero, one, or two equilibria. There are two distinct equilibria when the following inequality is satisfied:

$$
\bar{u}<(1-\alpha(\bar{v})) \varphi_{\max }(d)
$$

where $\varphi_{\max }(d)$ is the maximum value of the function $\varphi(z, d)$ with respect to $z$. Linearizing the model (1)-(3) at these equilibria, the eigenvalues satisfy

$$
\begin{aligned}
\lambda_{1} & =-T_{f}^{-1} \\
\lambda_{2}+\lambda_{3} & =-\varphi_{z}(\bar{z}, d)-T_{r}^{-1} \\
\lambda_{2} \lambda_{3} & =T_{r}^{-1} \varphi_{z}(\bar{z}, d)(1-\alpha(\bar{v}))
\end{aligned}
$$

where $\varphi_{z}$ denotes the partial derivative of $\varphi$ with respect to $z$. From (9)-(10), we conclude that the stability of the equilibria is determined by the sign of $\varphi_{z}(\bar{z}, d)$. The equilibrium is exponentially stable if $\varphi_{z}(\bar{z}, d)>0$, whereas it is unstable if $\varphi_{z}(\bar{z}, d)<0$. In Fig. 4 , the stable situation corresponds to equilibria to the left of the maximum of the curve, while unstable equilibria are located to the right of the maximum. When $\varphi_{z}(\bar{z}, d)=0$, one of the eigenvalues is zero and the stability of the equilibrium is determined by the center manifold dynamics

$$
\dot{z}=-\varphi(z, d)+\bar{u}+\bar{y}_{r} .
$$

The equilibrium $z=\bar{z}$ of (11) is unstable since $(z-\bar{z}) \dot{z}>0$ for $z>\bar{z}$.

\section{The Plugging Phenomenon}

The plugging phenomenon manifests itself under the form of a dramatic decrease of the production and an irreversible accumulation of material in the mill due to intermittent disturbances of the inflow rate and variations of clinker hardness.

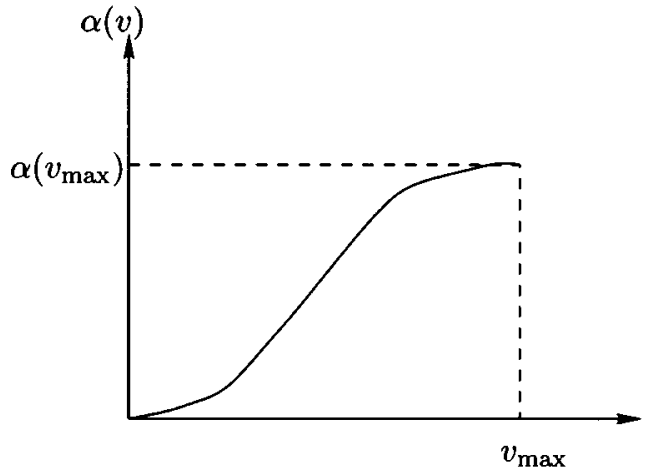

Fig.3. Separation function.

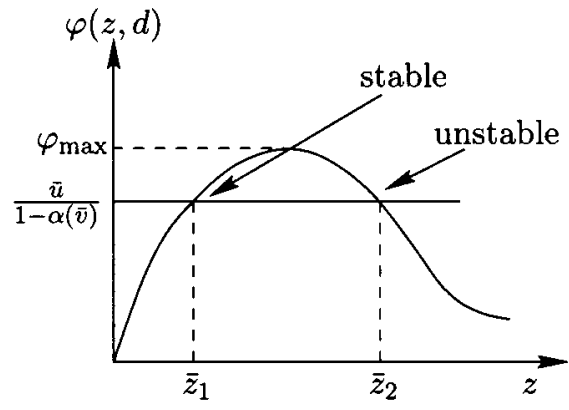

Fig. 4. Equilibria and their stability.

In the model (1)-(3) with constant inputs $\bar{u}$ and $\bar{v}$, plugging is a global instability which occurs as soon as the state $\left(y_{f}, y_{r}, z\right)$ enters the set $\Omega$ defined by the following inequalities (see Fig. 5):

$$
\begin{aligned}
& y_{f} \geq 0, \quad y_{r} \geq 0, \quad z \geq 0 \\
& (1-\alpha(\bar{v})) \varphi(z, d)<y_{f}<\bar{u} \\
& \alpha(\bar{v}) \varphi(z, d)<y_{r} \\
& \varphi_{z}(z, d)<0 .
\end{aligned}
$$

Indeed, it is not difficult to observe that $\Omega$ is a positively invariant set and that in $\Omega$

$$
\begin{aligned}
T_{f} \dot{y}_{f} & <0 \\
T_{r} \dot{y}_{r} & <0 \\
\dot{z} & >0 .
\end{aligned}
$$

Therefore, we have that in $\Omega$

$$
y_{f} \rightarrow 0, \quad y_{r} \rightarrow 0, \quad z \rightarrow \infty \text { as } t \rightarrow \infty .
$$

Hence, the level $z$ of material in the mill is accumulated without limitation while the production rate $y_{f}$ goes to zero.

\section{Robust Global StabiLIZATION}

The control objective considered in this paper is to regulate the production rate $y_{f}$ and the mill load $z$ at desired set points $y_{f}^{*}$ and $z^{*}$ by acting on the feed rate $u$ and the separator speed $v$. The controller must prevent the mill from plugging and achieve global stabilization. Moreover, the controller must be robust against modeling uncertainties. More precisely, our design only relies on the shape of the grinding function $\varphi(z, d)$ and the separation function $\alpha(v)$ as illustrated in Figs. 2 

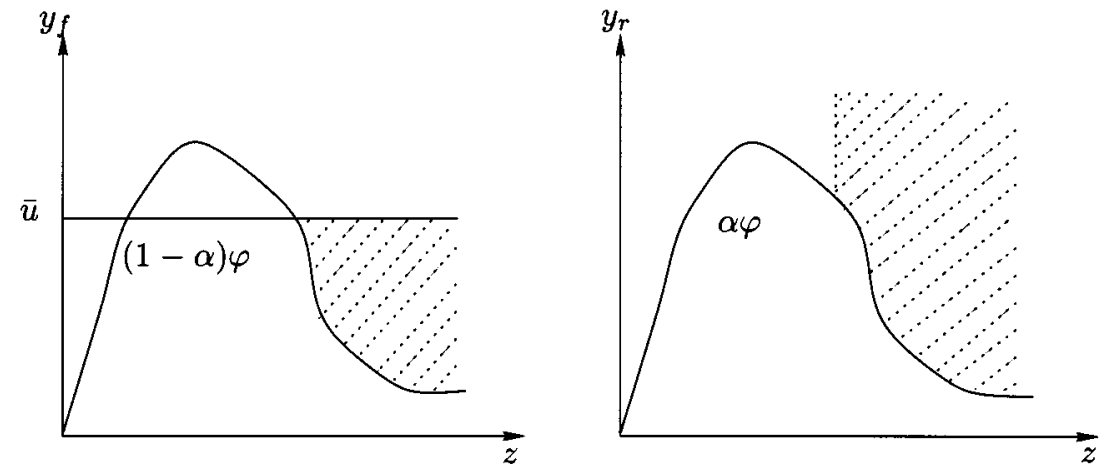

Fig. 5. The plugging set $\Omega$.
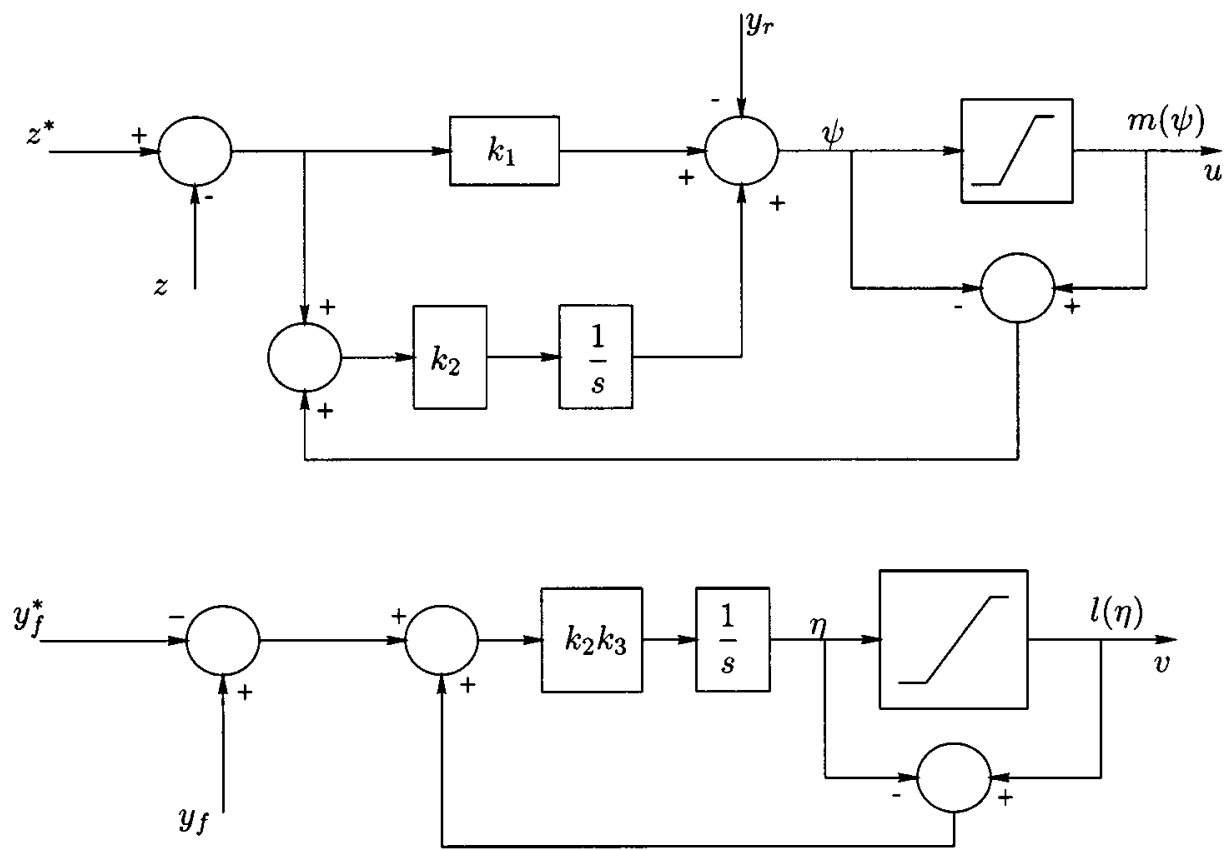

Fig. 6. Block diagram of the controller.

and 3. No quantitative analytical model of these functions is assumed to be known.

The control inflow rate $u$ and the control separation speed $v$ are physically constrained to be positive and saturated. The control objective will be achieved with the following control law:

$$
\begin{aligned}
u & =m(\psi) \\
\psi & =-y_{r}+k_{1}\left(z^{*}-z\right)+\theta \\
\dot{\theta} & =k_{2}\left(z^{*}-z\right)+k_{2}(m(\psi)-\psi) \\
v & =l(\eta) \\
\dot{\eta} & =k_{3}\left(k_{2}\left(y_{f}-y_{f}^{*}\right)+k_{2}(l(\eta)-\eta)\right)
\end{aligned}
$$

where $m(\psi)=\operatorname{sat}_{\left[0, u_{\max }\right.}(\psi)$ and $l(\eta)=\operatorname{sat}_{\left[0, v_{\max }\right]}(\eta)$.

This is a very simple control law made up of two saturated PI controllers with anti wind-up and a "feedforward" of $y_{r}$ into the $z$-regulator, which decouples the two loops (see Fig.6).

The closed-loop system (1)-(3), (13)-(17) is then rewritten as

$$
\begin{aligned}
T_{f} \dot{y}_{f} & =-y_{f}+(1-\alpha(v)) \varphi(z, d) \\
T_{r} \dot{y}_{r} & =-y_{r}+\alpha(v) \varphi(z, d) \\
\dot{z} & =-\varphi(z, d)+y_{r}+m(\psi)
\end{aligned}
$$

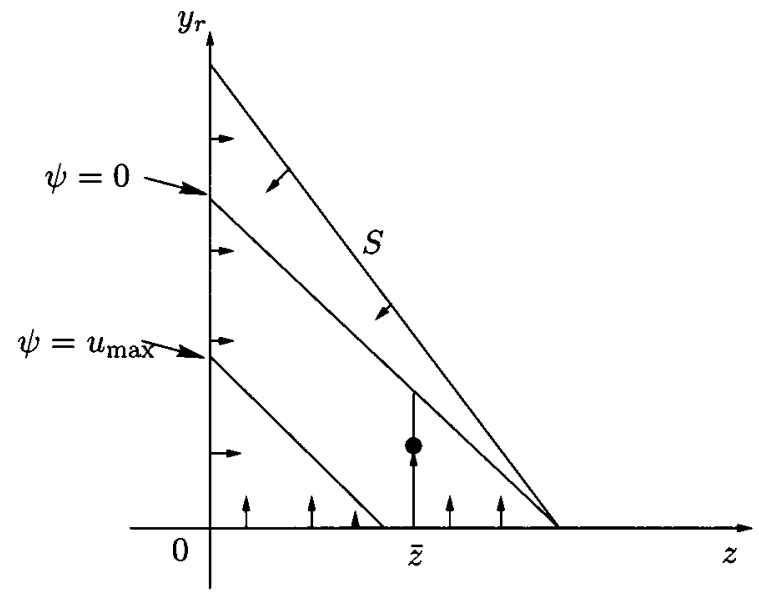

Fig.7. Invariant triangle $T$.

$$
\begin{aligned}
\dot{\theta} & =k_{2}\left(z^{*}-z\right)+k_{2}(m(\psi)-\psi) \\
\dot{\eta} & =k_{3}\left(k_{2}\left(y_{f}-y_{f}^{*}\right)+k_{2}(l(\eta)-\eta)\right) \\
\psi & =-y_{r}+k_{1}\left(z^{*}-z\right)+\theta \\
v & =l(\eta) .
\end{aligned}
$$



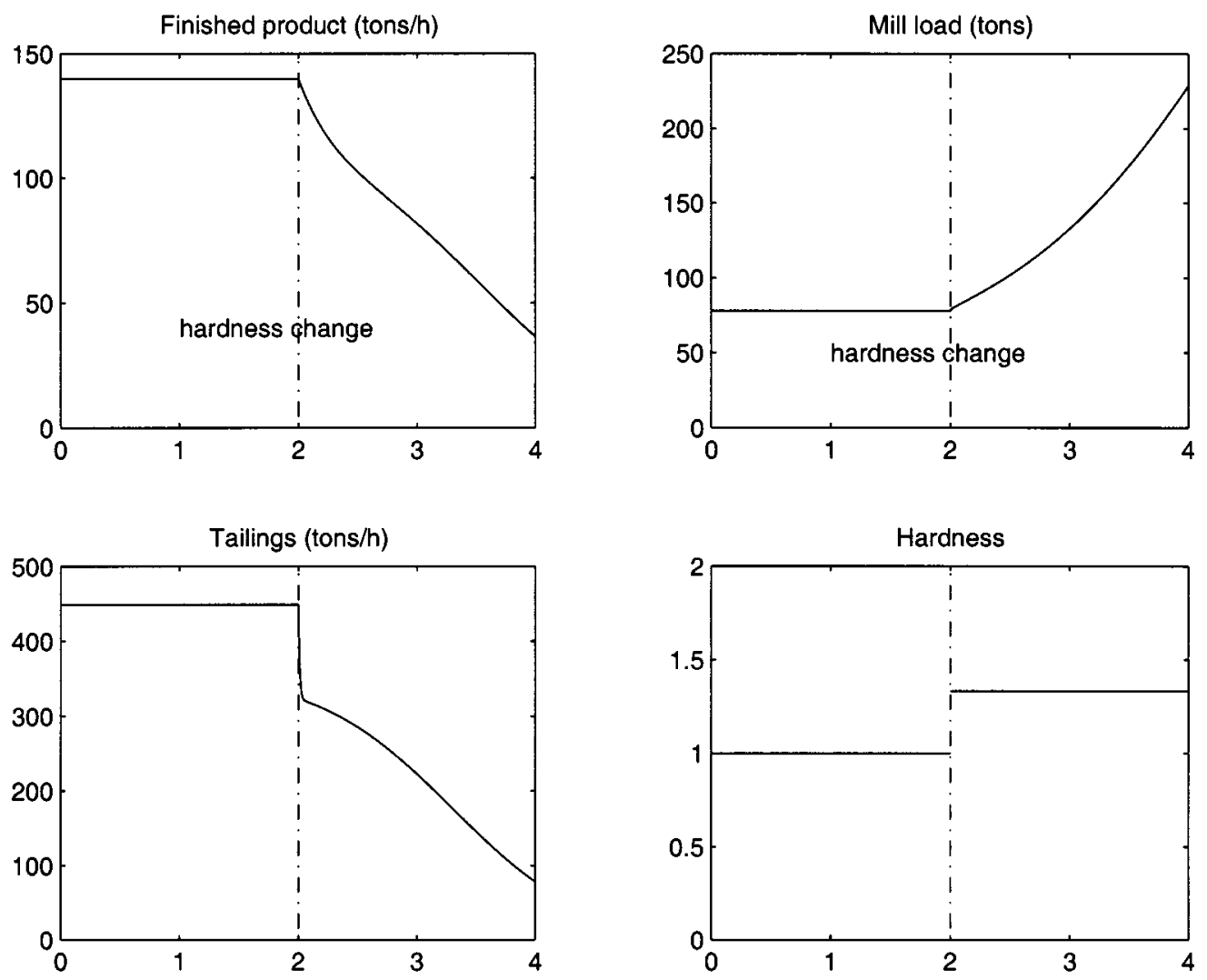

Fig. 8. The plugging phenomenon.

In the next theorem, the stability properties of this closed loop system will be analyzed. Some technical notations must be introduced. The clinker hardness $d$ is assumed to belong to an interval of admissible values $\left[d_{1}, d_{2}\right]$. For each value of $d \in\left[d_{1}, d_{2}\right]$, the grinding function $\varphi(z, d)$ as depicted in Fig. 2 is a twice differentiable Lipschitz function of $z$, with a Lipschitz constant denoted $k_{0}(d)$. For each value of $z \in$ $\mathbb{R}^{+}, \varphi(z, d)$ is a decreasing function of $d$. The maximal value of $\varphi$ is denoted

$$
\varphi_{\max }=\max _{z \in \mathbb{R}^{+}} \varphi\left(z, d_{1}\right) .
$$

The separation function $\alpha(v)$ as represented in Fig. 3 is also twice differentiable with

$$
\alpha(0)=\alpha^{\prime}(0)=\alpha^{\prime \prime}(0)=\alpha^{\prime}\left(v_{\max }\right)=\alpha^{\prime \prime}\left(v_{\max }\right)=0 .
$$

Then, we have the following stability result where it is shown that the closed loop system has a unique equilibrium which is semiglobally stable in the domain of physical existence of the process.

Theorem: If $d \in\left[d_{1}, d_{2}\right], u_{\max } \rightarrow \varphi_{\max }$, $k_{1}>\max \left(1, \max _{d \in\left[d_{1}, d_{2}\right]} k_{0}(d)\right), k_{3}>0$. Then, for any compact sets $\mathcal{C}_{(\theta, \eta)} \subset\left[-k_{1} z^{*}, \rightarrow\left[\times \mathbb{R}\right.\right.$ and $\mathcal{C}_{\left(y_{f}, y_{r}, z\right)} \subset \mathbb{R}_{+}^{3}$, there exists $k_{2}^{*}>0$ such that for all $0<k_{2}<k_{2}^{*}$ the single equilibrium of the closed-loop system (18)-(24) is asymptotically stable and its region of attraction contains $\mathcal{C}_{\left(y_{f}, y_{r}, z\right)} \times \mathcal{C}_{(\theta, \eta)}$.

Proof: The domain $\mathcal{A}=\left\{\left(y_{f}, y_{r}, z, \theta, \eta\right) \mid y_{f} \geq 0, y_{r} \geq\right.$ $\left.0, z \geq 0, \theta \geq-k_{1} z^{*}, \eta \in \mathbb{R}\right\}$ is invariant. Indeed $y_{r}$ and $y_{f}$ are positive by nature, and $z$ is positive by construction of the control law. Moreover, if $\theta=-k_{1} z^{*}$, then $\psi$ is necessarily negative and the control is saturated at zero, which implies that

$$
\dot{\theta}=k_{2}\left(\left(k_{1}-1\right) z+y_{r}+z^{*}\right)>0 \text {. }
$$

We may, therefore, assume that $\theta$ is initialized such that $\theta \geq-k_{1} z^{*}$ and restrict our attention to the behavior of the system inside the subdomain $\mathcal{A}$.

The special case without integral action is first considered because it is the reduced-order model for a subsequent singular perturbation analysis.

1) Stability Proof Without Integral Action: Let us first consider the case without integral action (i.e., $k_{2}=0$ ) and with the variables $\theta$ and $\eta$ fixed at arbitrary values $\bar{\theta} \geq-k_{1} z^{*}$ and $\bar{\eta} \in \mathbb{R}$. The closed-loop system (18)-(24) reduces to

$$
\begin{aligned}
T_{f} \dot{y}_{f} & =-y_{f}+(1-\alpha(v)) \varphi(z, d) \\
T_{r} \dot{y}_{r} & =-y_{r}+\alpha(v) \varphi(z, d) \\
\dot{z} & =-\varphi(z, d)+y_{r}+m(\psi) \\
\psi & =-y_{r}+k_{1}\left(z^{*}-z\right)+\bar{\theta} \\
v & =l(\bar{\eta}) .
\end{aligned}
$$

Any equilibrium $\left(\bar{y}_{f}, \bar{y}_{r}, \bar{z}\right)$ must satisfy

$$
\begin{aligned}
& 0=-\bar{y}_{f}+(1-\alpha(l(\bar{\eta}))) \varphi(\bar{z}, d) \\
& 0=-\bar{y}_{r}+\alpha(l(\bar{\eta})) \varphi(\bar{z}, d) \\
& 0=-\varphi(\bar{z}, d)+\bar{y}_{r}+m\left(-\bar{y}_{r}+k_{1}\left(z^{*}-\bar{z}\right)+\bar{\theta}\right)
\end{aligned}
$$

Eliminating $\bar{y}_{r}$ in (33) and (34), we obtain

$(\alpha(l(\bar{\eta}))-1) \varphi(\bar{z}, d)+m\left(-\alpha(l(\bar{\eta})) \varphi(\bar{z}, d)+k_{1}\left(z^{*}-\bar{z}\right)+\bar{\theta}\right)$ $=0$.

This equation has no solution $\bar{z}$ when $\left(-\alpha(l(\bar{\eta})) \varphi(\bar{z}, d)+k_{1}\left(z^{*}-\right.\right.$ $\bar{z})+\bar{\theta})>u_{\max }$ (because $u_{\max }>\varphi_{\max }$ ) or $<0$ (because this would require $\bar{z}=0$, and the argument of $m$ is $\bar{\theta}+k_{1} z^{*} \geq 0$ when $\bar{z}=$ 

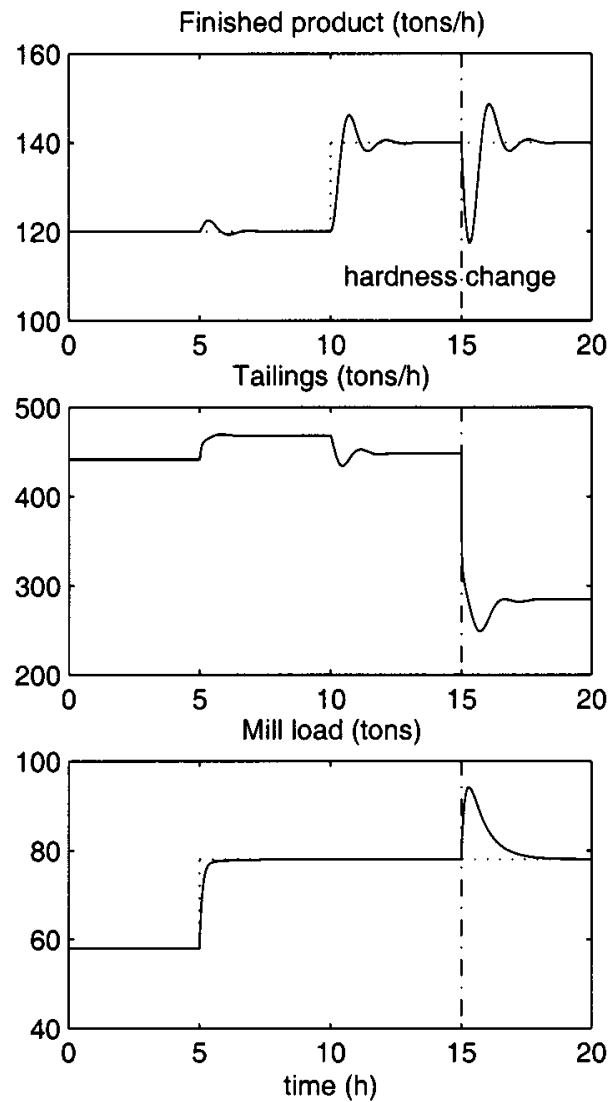

Fig. 9. Simulation of the closed-loop system.

0 , which is a contradiction). Hence, solutions must lie in the region without saturation and satisfy

$$
\varphi(\bar{z}, d)+k_{1} \bar{z}=k_{1} z^{*}+\bar{\theta}
$$

The left-hand side is a monotonic increasing function of $\bar{z}$ [because $k_{1}>k_{0}(d)$ ] with value 0 at $\bar{z}=0$. The right-hand side is strictly positive. Therefore, this equation has a unique solution $\bar{z}>0$. The unique corresponding equilibrium values $\left(\bar{y}_{f}, \bar{y}_{r}\right)$ follow from (32) to (33).

We have just shown that a unique equilibrium exists for any value of $\bar{\theta} \geq-k_{1} z^{*}$ and $\bar{\eta} \in \mathbb{R}$, thus obtaining a family of equilibria $\left(\bar{y}_{f}(\bar{\theta}, \bar{\eta}), \bar{y}_{r}(\bar{\theta}, \bar{\eta}), \bar{z}(\bar{\theta})\right)$ parametrized by $\bar{\theta}$ and $\bar{\eta}$. These functions $\bar{y}_{f}(\bar{\theta}, \bar{\eta}), \bar{y}_{r}(\bar{\theta}, \bar{\eta})$, and $\bar{z}(\bar{\theta})$ are $\mathcal{C}^{2}$ because $\varphi(z, d)$ and $\alpha(v)$ are $\mathcal{C}^{2}$ and because of (25).

We still have to show that $\left(\bar{y}_{f}(\bar{\theta}, \bar{\eta}), \bar{y}_{r}(\bar{\theta}, \bar{\eta}), \bar{z}(\bar{\theta})\right)$ is a globally asymptotically stable (GAS) and locally exponentially stable (LES) equilibrium of the system (27)-(31) for any fixed value of $(\bar{\theta}, \bar{\eta})$.

The system (27)-(31) is the cascade of the system (28)-(31) with the globally exponentially stable (GES) scalar subsystem

$$
T_{f} \dot{y}_{f}=-y_{f}+(1-\alpha(v)) \varphi(z, d) .
$$

We first show that the equilibrium of the subsystem (28)-(31) is GAS-LES for any $(\bar{\theta}, \bar{\eta})$. Let us select the constant $c>0$ large enough such that

$$
\psi=-y_{r}-k_{1} z+k_{1} z^{*}+\bar{\theta}<0
$$
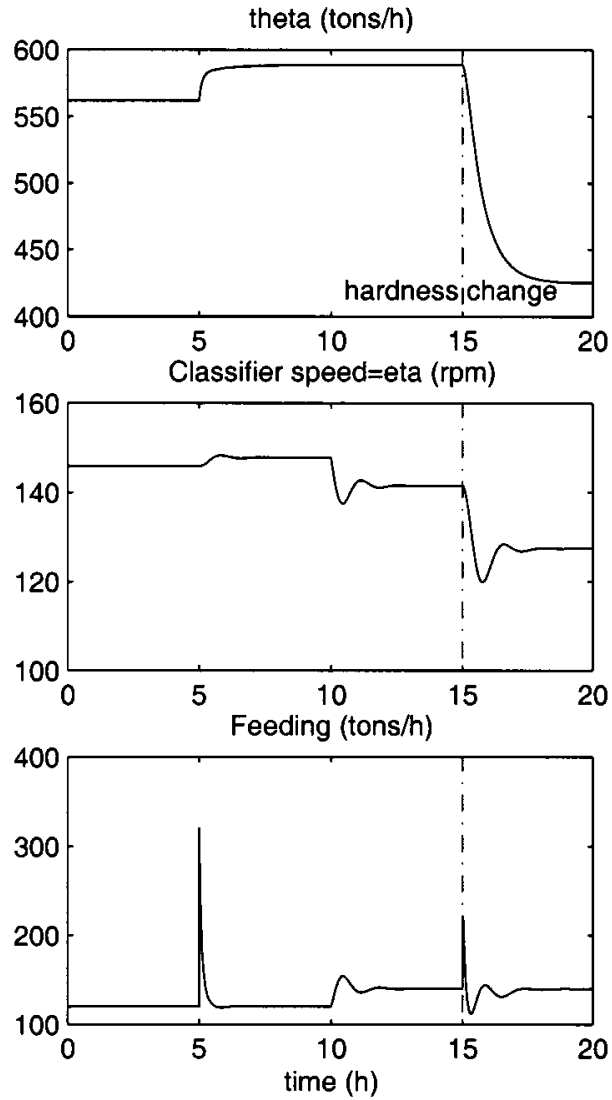

for all $\left(y_{r}, z\right)$ in the set

$$
S=\left\{\left(y_{r}, z\right): y_{r} \geq 0, z \geq 0, T_{r} y_{r}+z=c\right\}
$$

Since $\dot{z}+T_{r} \dot{y}_{r}<0$ when $\psi<0$, the triangle $T$ bounded by the coordinate axes and the straight line $S$ is invariant and globally attractive (see Fig.7).

All the subsystem trajectories are bounded and reach $T$ in finite time. The limit set of any trajectory inside $T$ is either the equilibrium $\left(\bar{y}_{r}(\bar{\theta}, \bar{\eta}), \bar{z}(\bar{\theta})\right)$ or a limit cycle enclosing the equilibrium. The segment of straight line joining the points $(0, \bar{z}(\bar{\theta}))$ and $\left(\bar{y}_{r}(\bar{\theta}, \bar{\eta})\right.$, $\bar{z}(\bar{\theta}))$ is invariant. Because any limit cycle should cross this segment, the only possible limit set is the equilibrium which is, thus, GAS. From the Jacobian linearization of the system, the equilibrium is also LES. Now, the equilibrium of equation (18) is GES when $\left(y_{r}, z\right)=\left(\bar{y}_{r}(\bar{\theta}, \bar{\eta}), \bar{z}(\bar{\theta})\right)$. It follows from [8, Th. A] that the whole subsystem (18)-(24) is GAS-LES.

2) Stability Proof with Integral Actions: We now come back to the case where $k_{2} \neq 0$ and there is an integral action in the loop. We will establish semiglobal stabilizability when $\theta$ and $\eta$ are slowly varying. This can be achieved by taking $k_{2}=\epsilon$ small enough. With the change of time scale $\tau=\epsilon t$, the system is rewritten in a two-time scale form as follows:

$$
\begin{aligned}
\dot{\theta} & =z^{*}-z+m(\psi)-\psi \\
\dot{\eta} & =k_{3}\left(y_{f}-y_{f}^{*}\right)+k_{3}(l(\eta)-\eta) \\
\epsilon T_{f} \dot{y}_{f} & =-y_{f}+(1-\alpha(v)) \varphi(z, d) \\
\epsilon T_{r} \dot{y}_{r} & =-y_{r}+\alpha(l(\eta)) \varphi(z, d) \\
\epsilon \dot{z} & =-\varphi(z, d)+y_{r}+m(\psi)
\end{aligned}
$$




$$
\psi=-y_{r}+k_{1}\left(z^{*}-z\right)+\theta
$$

The fast subsystem (39)-(41) is the system which has been analyzed above, without integral action. When $\epsilon=0$, the fast variables are at their equilibrium $\left(\bar{y}_{f}(\theta, \eta), \bar{y}_{r}(\theta, \eta), \bar{z}(\theta)\right)$. The slow subsystem (37), (38) then reduces to

$$
\begin{aligned}
& \dot{\eta}=k_{3}\left((1-\alpha(l(\eta))) \varphi(\bar{z}(\theta), d)-y_{f}^{*}+l(\eta)-\eta\right) \\
& \dot{\theta}=z^{*}-\bar{z}(\theta)+m(\psi)-\psi .
\end{aligned}
$$

Since $z$ can reach an equilibrium only when $m(\psi)=\psi$, (44) becomes

$$
\dot{\theta}=z^{*}-\bar{z}(\theta)=\frac{\varphi(\bar{z}(\theta), d)-\theta}{k_{1}}
$$

and the equilibrium $\theta=\varphi\left(z^{*}, d\right)$ of (45) is GES. Now, (43) with $\theta=\varphi\left(z^{*}, d\right)$ and $z=\bar{z}\left(\varphi\left(z^{*}, d\right)\right)=z^{*}[\operatorname{see}(35)]$ is

$$
\dot{\eta}=k_{3}\left((1-\alpha(l(\eta))) \varphi\left(z^{*}, d\right)-y_{f}^{*}\right)+k_{3}(l(\eta)-\eta) .
$$

This equation has a unique equilibrium $\eta^{*}$. It is easily seen that $\dot{\eta}<$ 0 when $\eta>\eta^{*}$ and $\dot{\eta}>0$ when $\eta<\eta^{*}$ and consequently that the equilibrium $\eta^{*}$ is GAS-LES. It follows from [8, Th. 8.1] that the equilibrium of the slow subsystem (37), (38) is GAS-LES.

The proof is completed by applying the singular perturbation analysis in [6, Th. 3.18]. For any compact sets $\mathcal{C}_{(\theta, \eta)} \subset\left[-k_{1} z^{*}, \rightarrow[\times \mathbb{R}\right.$ and $\mathcal{C}_{\left(y_{f}, y_{r}, z\right)} \subset \mathbb{R}_{+}^{3}$, there exists $k_{2}^{*}>0$ such that for all $0<k_{2}<$ $k_{2}^{*}$ the equilibrium of system (18)-(23) is asymptotically stable and its region of attraction contains $\mathcal{C}_{(\theta, \eta)} \times \mathcal{C}_{\left(y_{f}, y_{r}, z\right)}$. Q.E.D.

\section{SimUlation RESULTS}

The effectiveness of the proposed control law has been assessed through simulations where the model (1)-(3) represents the plant with analytical forms for the $\varphi$ and $\alpha$ functions which satisfy the shape assumptions given in Sections II and V:

$$
\begin{aligned}
\varphi(z, d) & =20 z \exp \left(-\frac{d z}{80}\right) \\
\alpha(v) & =9\left(\frac{v}{v_{\max }}\right)^{3}-13.5\left(\frac{v}{v_{\max }}\right)^{4}+5.4\left(\frac{v}{v_{\max }}\right)^{5} \\
v_{\max } & =200, \quad \alpha_{\max }=0.9
\end{aligned}
$$

and the time constants $T_{f}=0.3[\mathrm{~h}], T_{r}=0.01[\mathrm{~h}]$

These functions have been tuned in order to match experimental step responses of an industrial cement grinding circuit (see [4]).

The plugging phenomenon is first illustrated in Fig. 8. The mill is initially running at a stable steady state $\bar{z}=78, \bar{y}_{f}=140, \bar{y}_{r}=$ $448.4, \bar{u}=140, \bar{v}=141.5, d=1$ during the first two hours. At time $t=2$ hours, a small change of clincker hardness $(d=1.33)$ occurs. This disturbance produces plugging in the mill, as it can be seen in the figure, with an exponential unbounded accumulation of the mill load while the two other state variables tend to zero as expected.

Various aspects of the closed-loop behavior are then illustrated in Fig. 9 with the design parameters set to $k_{1}=10, k_{2}=10, k_{3}=0.2$. During the first six hours, the mill is at the equilibrium with $y_{f}^{*}=120$, $z^{*}=58$ and $d=1$. A step change is then introduced on the mill load setpoint $\left(z^{*}=78\right)$ at $t=5$ hours. This is followed by a step change on the finished product setpoint $\left(y_{f}^{*}=140\right)$ at $t=10$ hours and by a hardness change $(d=1.33)$ at $t=15$ hours. Note that this hardness change is identical to that of Fig. 8 and would destabilize the mill in open loop. Furthermore, the same hardness change would also produce a destabilization of the $L Q G$ controller described in [1].

The following comments can be drawn from these figures.

1) The mill level is fairly well decoupled from finished product setpoint variations. This is mainly due to the feedforward compensation present in (14).

2) The rejection of the hardness change disturbance by the mill load controller is very fast, which indeed prevents the mill from going into plugging. The effect of hardness changes on the finished product takes a longer time to disappear, but it is also less critical since it does not destabilize the plant.

\section{CONCLUSION}

The goal of this note was essentially to show that it is effectively possible to fully prevent industrial mills from plugging by state feedback, provided it includes the mill load. In [5], the authors had also observed that this state variable is essential. Stability was, therefore, the main issue of this note. Obviously, many other issues, for instance, the limits of performance imposed by the stability requirement or the optimal selection of set points for productivity, are interesting and should be investigated.

\section{REFERENCES}

[1] V. Van Breusegem, L. Chen, V. Werbrouck, G. Bastin, and V. Wertz, "Multivariable linear quadratic control of a cement mill: An industrial application," Control Eng. Prac., vol. 2, no. 4, pp. 605-611, 1994.

[2] I. K. Craig and I. M. MacLeod, "Robust controller design and implementation for a run-of-mine ore milling circuit," Control Eng. Prac., vol. 4, no. 1, pp. 1-12, 1996.

[3] D. G. Luenberger, Introduction to Dynamic Systems. New York: Wiley, 1979.

[4] L. Magni, G. Bastin, and V. Wertz, "Multivariable nonlinear predictive control of cement mills," IEEE Trans. Contr. Syst. Technol., vol. 7, pp. 361-371, July 1999.

[5] M. Boulvin, C. Renotte, A. Vande Wouwer, M. Remy, L. Tarasiewics, and P. César, "Modeling, simulation, and evaluation of control loops for a cement grinding process," in Proc. European Control Conf. (CDROM), Brussels, Belgium, July 1997, Paper TH-E-H4.

[6] R. Sepulchre, M. Jankovic, and P. Kokotovic, Constructive Nonlinear Control. New York: Springer-Verlag, 1997.

[7] M. Vidyasagar, Nonlinear Systems Analysis. Englewood Cliffs, NJ: Prentice-Hall, 1993.

[8] F. Viel, F. Jadot, and G. Bastin, "Robust feedback stabilization of chemical reactors," IEEE Trans. Automat. Contr., vol. 42, pp. 473-481, Apr. 1997. 